\title{
Lhasa de Sela et la traversée des espaces
}

\author{
Robert ProulX (Wolfville, CA)
}

\section{Summary}

The singer-songwriter Lhasa de Sela (1972-2010) launched her career and produced her three records in Montreal where she arrived in 1991. Not only did she change the face of migrant song in Quebec, but she also enjoyed international success, embarking on long world tours and selling more than a million records. This analysis will focus on the songs from her second album, The Living Road, and will show that Lhasa de Sela transcended linguistic and artistic frontiers by crossing the geographical border when she made Montreal her home and creative hub.

L'auteure-compositrice-interprète d'origine américano-mexicaine Lhasa de Sela (19722010) a lancé sa carrière et produit ses trois disques à Montréal où elle était arrivée en 1991. Celle dont on a dit qu'elle " avait changé le visage de la chanson immigrante au Québec » (Bernard 2010, A1) a connu un succès mondial, faisant de longues tournées internationales et vendant plus d'un million de disques au Québec, en France, en Allemagne, au Canada, au Japon et aux États-Unis. Après avoir donné quelques éléments biographiques, essentiels dans le cas de cette artiste migrante, notre analyse se concentrera sur les chansons de son deuxième album au titre éloquent, The Living Road, afin de montrer que Lhasa de Sela a transcendé les frontières linguistiques et artistiques en traversant la frontière géographique pour faire de Montréal son port d'attache et son lieu de création.

\section{La traversée des espaces : repères biographiques}

Lhasa de Sela naît le 27 septembre 1972 dans un petit village de l'État de New York. Elle est la fille du Mexicain Alejandro Sela, poète et professeur d'espagnol, et de l'Américaine Alexandra Karam, actrice et photographe, qui est d'origine russo-polonaise par sa mère et libanaise par son père. Pendant son enfance, sa famille sillonne les États-Unis et le Mexique à bord d'un autobus. C'est la mère qui fait l'école aux enfants, et le soir ils lisent, chantent ou font de la musique, car ces artistes nomades n’ont pas la télévision. Sa famille, très déra- 
cinée, lui fait dire : "Le déracinement, c’est mon pays. Mes ancêtres ont tous des origines différentes. » (Lévesque, 2003) Elle ajoute :

Mes aïeux sont venus de Pologne, d'Espagne, d'Ukraine, du Panama, du Liban, d'Écosse, d'Angleterre, d'Algérie, du Mexique. C'étaient des gens qui bougeaient, qui laissaient tout, qui se mariaient avec des gens d'autres pays. C'étaient des personnes bilingues, trilingues. Chrétiens, juifs, musulmans, athées. Ambassadeurs, inventeurs, fermiers, soldats, actrices, rabbins, industriels, musiciennes, couturières. (De Sela 2008, 33)

À l'âge de 13 ans, après le divorce de ses parents, Lhasa commence à chanter du jazz dans un café grec de San Francisco. À 18 ans, elle étudie la culture de la Grèce antique au St. John's College de Santa Fe, un programme qu'elle abandonne en mars 1991. Elle débarque alors à Montréal pour rendre visite à ses sœurs qui étudiaient à l'École nationale de cirque. Elle travaille comme serveuse à la Maison de la culture mondiale du boulevard Saint-Laurent et se produit sur scène le soir, interprétant des chansons de Billie Holiday et de la ranchera mexicaine Chavela Vargas, les deux pôles de «son identité morcelée » (Bernard 2010, A1). C'est dans la métropole québécoise qu'elle fait une rencontre déterminante, celle d'Yves Desrosiers, avec qui elle crée son premier album en 1997, et le résultat de leur collaboration produira La llorona, entièrement en espagnol, qui connaîtra un succès mondial : plus de 700000 disques vendus en plus d'un prix Félix au Québec dans la catégorie «musique du monde » en 1997 et, en 1998, un prix Juno au Canada pour Best Global Artist.

Encensé par ses fans, La llorona est devenu un classique de musique du monde en quelques années. La llorona ("La pleureuse ») évoque une "Amérique latine à la fois réelle et imaginaire, née de la mémoire d'une enfance itinérante, et l'album n'aurait sans doute pas pu voir le jour ailleurs qu'à Montréal, où il a été écrit, en plein cœur de l'hiver " (Lhasa de Sela, site web). Et dans une interview qu'elle accorde au magazine québécois L'Actualité, elle confie :

Plus le temps passe, plus je découvre des choses à aimer ici. J'ai une très grande affection pour le Québec. C’est... [silence] ici que j'ai connu ce qui se rapproche le plus du sentiment d'avoir un pays. (Chartrand 1998, 24)

À la suite de ce succès, elle part en tournée en Europe et en Amérique du Nord en 1998 et 1999, puis déménage en France pour rejoindre ses sœurs qui travaillent alors pour le cirque contemporain Pocheros. Lhasa s'établit ensuite à Marseille pendant deux ans et demi, où elle recommence à écrire. Mais c'est de retour à Montréal en 2002 qu'elle finalise son deuxième album. Alors que La llorona est écrit et chanté entièrement en espagnol, The Living Road (2003) est écrit en français, en anglais et en espagnol. Il apporte à Lhasa une reconnaissance encore une fois générale. Ses interprétations font l'unanimité et " on la reconnaît comme une enfant du pays » (Lhasa de Sela, site web) un peu partout dans le monde. L'album figure 
d'ailleurs en troisième place des meilleurs albums de musique du monde sortis durant les années 2000 du Times de Londres, et Lhasa a été récompensée au titre de "Meilleure artiste des Amériques » lors du gala des World Music Awards de la BBC en 2005.

Son troisième album, auquel elle donne son nom, est sorti en 2009. Lhasa est écrit complètement en anglais, sa langue maternelle, mais il est encore une fois enregistré à Montréal. Elle lance son album au théâtre Corona de Montréal en avril et le présente au public parisien au théâtre des Bouffes-du-Nord dans un époustouflant concert d'une heure en mai. Après deux concerts en Islande fin mai, la tournée qui annonçait son grand retour sur scène à l'automne 2009 est annulée pour des raisons de santé. On apprendra par la suite qu'elle a combattu un cancer du sein pendant plus de 21 mois, et elle meurt le soir du $1^{\text {er }}$ janvier 2010 dans sa résidence de Montréal. La nouvelle de son décès bouleverse et chagrine la communauté artistique et ses nombreux fans comme l'exprime le journaliste Alexandre Vigneault de La Presse dans son article intitulé "Lhasa de Sela, l'étoile filante ":

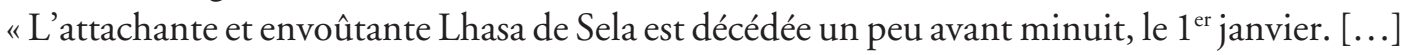
Son départ prématuré laisse dans le deuil une imposante famille élargie, des fans disséminés sur plusieurs continents, ainsi que de nombreux amis musiciens ». (Vigneault 2010, A1)

\section{Les traversées symboliques dans The Living Road}

Cet article va montrer comment la migration a influencé l'œuvre de cette artiste. Ceci se reflète, en particulier, par la présence de chansons écrites en français et en anglais sur son deuxième disque The Living Road (2003) et par une plus grande variété de styles musicaux et d'instruments, ce qui indique le désir qu'elle a toujours eu d'explorer de nouvelles voies musicales et thématiques. D'ailleurs, on trouve ici de nouvelles textures musicales comme des musiques de cirque à la Nino Rota, ce en quoi Lhasa a probablement été influencée par l'univers de ses sœurs qu'elle a retrouvées, d'abord à l'École nationale de cirque de Montréal, puis en France.

Ce qui frappe chez cette artiste unique, c'est l'effet que la migration a eu sur elle et, par conséquent, sur sa carrière ; c'est comme si le fait d'émigrer avait été pour elle le catalyseur qui avait fait exploser sa créativité. En fait, les rencontres déterminantes avec des musiciens québécois comme Yves Desrosiers, Jean Massicotte et François Lalonde ont agi comme éléments déclencheurs et permis l'éclosion de son talent et le démarrage de sa carrière. Et, en apprenant une nouvelle langue, le français, grâce à son cercle d'amis montréalais, elle a pu et a osé créer dans ce nouvel idiome, ce qui est tout à fait remarquable. D’ailleurs, elle l'exprime elle-même dans son livre La route chante qui relate en mots et en images ses souvenirs d'une enfance itinérante et son amour de la musique : "À force de vivre à Montréal, d'être partie et revenue, je me rends compte que cette ville est très précieuse pour moi. Et qu'il y avait bien une raison pour laquelle j'étais là. C'est ma terre fertile où ce grain peut pousser. »(De Sela 2008, 37) 
Nous nous attarderons à ses chansons en français et en anglais tout en les comparant avec les autres écrites en espagnol, sa langue paternelle. De toute façon, et cela aussi est exceptionnel, elle a elle-même traduit ses chansons en anglais et en français. Enfin, qu'elles soient en espagnol, en anglais ou en français, on y retrouve ses thèmes de prédilection qui sont, précisément, le voyage et la migration, assortis d'une quête de la rencontre de l'Autre qui passe par l'amour. D'ailleurs, ce thème de l'amour est lui aussi traité comme un mouvement, une rencontre, souvent difficile et houleuse entre deux êtres, faite d'avancées et de reculs. Elle lance d'ailleurs un soir au public du Club Soda à Montréal : " Je chantais des amours malheureuses, mais je n'en avais jamais vécues. Alors, je me suis dit qu'il fallait que je vive cette expérience pour bien la chanter. J'ai donc trouvé la personne parfaite pour me rendre malheureuse! » (Chartrand 1998, 24)

Un examen sommaire du lexique est fort révélateur des thématiques récurrentes dans son œuvre : on trouve partout des verbes comme ' $s$ 'en aller , ‘ voyager ’, ‘ arriver ’, ‘ revenir ',

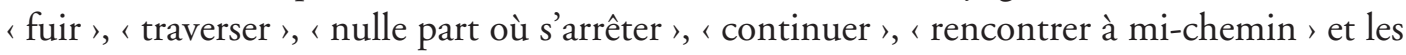

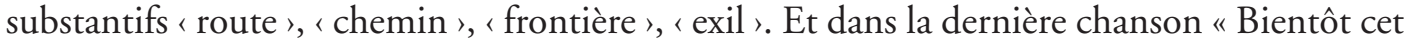
espace sera trop petit » de l'album The Living Road, on trouve cette allusion directe au titre : " Je poserai mon pied sur la route vivante / Et je serai portée jusqu'au cœur du monde. »

Ces dernières phrases semblent jeter un doute sur son désir d'enracinement à Montréal. Est-ce à dire que malgré ce qu'elle avait exprimé sur l'importance que Montréal a eue dans sa vie et sa carrière, elle n'y serait probablement pas restée ? Effectivement, pour cette âme bohémienne, jurer de prendre racine pour de bon est impossible. Déjà en 1998, elle affirmait : " Je ne pense pas que je pourrai rester toute ma vie à la même place. Mais j'adore Montréal et j'y reviendrai toujours pour recharger mes batteries. " (Chartrand 1998, 24) Quoi qu'il en soit, c'est à Montréal qu'elle a posé ses valises de nomade le plus longtemps et qu'elle a créé au fil des rencontres avec ses amis musiciens une œuvre forte, multilingue et très originale qui a traversé les espaces et aboli les frontières entre les humains qu'elle a su faire vibrer aux émotions qui la transportaient.

La chanson "J'arrive à la ville » semble emblématique du trajet et surtout du projet de vie de Lhasa de Sela qui, pour être le sien, correspond aussi à celui d'autres migrants qui se déracinent d'un lieu pour s'enraciner ailleurs. Pour bien illustrer ceci, les quatre couplets de la chanson qui, par certains éléments répétés jouant aussi le rôle de refrains, débutent par les mots " Moi aussi », indiquent qu'il s'agit d'une expérience commune à d'autres migrants comme elle.

Chaque partie de la chanson donne les raisons du départ ou de l'arrivée à la ville, lesquelles sont multiples et différentes selon les cas. En effet, on vient à la ville pour " y verser sa vie ", pour y trouver refuge " en fuyant " la violence de la guerre, la discrimination, la pauvreté « les mains vides », bref pour y oublier un passé douloureux : « J'ai un passé / Mais je ne m'en sers pas / Le futur sera mieux / Tellement mieux que ça. » Le texte insiste sur l'abandon du passé avec l'image des " mains vides " et évoque habilement, et avec une économie de moyens remarquable, l'arrivée à Montréal des peuples des Premières Nations qui viennent du nord et celle des Haïtiens et Latino-Américains qui migrent du sud : «J'arrive les mains 
vides / Au sud du nord / Au nord du sud. » En tout cas, cette chanson présente sans équivoque la migration de manière positive comme le montre l'image du " géant qui monte dans la rue » et les propos de la narratrice qui répète à la $1^{\text {ìre }}$ et à la $4^{\text {ième }}$ strophe : "Je monte la rue / Comme un géant / Ça c'est la ville / Et ça ... / C'est ma vie. " Elle aurait pu écrire "C'est la ville et c'est ma ville ", mais cette formulation est encore plus forte, car elle révèle l'adaptation, l'intégration et l'amour qu'elle a pour Montréal, l'adéquation parfaite entre sa nouvelle vie et sa ville d'adoption. Dans une interview au Devoir en 2003, elle expliquait d'ailleurs : "Le passé est là, sans jamais expliquer tout. Le futur est présent à moi dans la mesure où je ne veux pas être un bateau à la merci des vagues. » (Lévesque 2003, B8) Dans ce même entretien, à l'époque de la sortie de son deuxième album, Lhasa disait aussi que "The Living Road c'est le lieu où se retrouvent la vie et le destin de chaque personne. Ce lieu a sa logique. Mon pays, c'est aussi la logique particulière de ma vie. [...] Aller loin tout en restant proche de soi, c'est cela qui m'intéresse. » (Lévesque 2003, B8)

Ces déclarations, qui peuvent sembler contradictoires, illustrent la tension continuelle entre l'enracinement et le déracinement, entre rester et partir qui tissent la trame de sa vie et celle du disque qu'elle a créé, "un album où elle chante en trois langues douze nouvelles chansons qui parlent de vertiges et de voyages, qui mettent le cœur en scène et affirment l'impérative nécessité d'avancer " (Lévesque 2003, B8). Cette idée, qui est au cœur de son œuvre, est présente dans plusieurs chansons, mais nulle part aussi clairement exprimée que dans « Nulle part sur cette route" :

Maintenant j'habite ce pays

On m'appelle par ce nom

Je parle ce langage

Ce n'est pas tout à fait pareil

Et seulement pour ces raisons

Ici c'est chez moi

Et les lieux desquels j'étais

Loin ont disparu

Dans son livre, intitulé La route chante, elle dit explicitement que " ces lieux disparus sont ceux du Mexique " (De Sela 2008, 36) où elle avait passé son enfance et son adolescence bohêmes. La deuxième strophe qui revient comme un refrain en finale est comme une exhortation à oublier le passé :

Tu as voyagé si longtemps

Il faut continuer

Ne regarde même pas en arrière

Pour voir ce que tu as traversé

Même si ton corps se courbe

Sous le fardeau 
Il n'y a aucun endroit où s'arrêter

Nulle part sur cette route

On est en droit de se demander si cette exhortation à faire table rase du passé et du chemin parcouru s'adresse à quelqu'un d'autre ou à elle-même puisque dans le milieu de la chanson c'est un < je > qui revient pour raconter ses déboires amoureux. L'amour y est décrit comme une prison, on y trouve la "peur ", la méfiance de l'un et le désir de l'Autre de se libérer des affres de l'amour ; "envies et désirs ardents " pour ne plus être " avalée par les ténèbres ".

Cette même idée d'effacer le passé se trouve dans «La frontera " «La frontière »), mais avec la nuance que, parfois, ce cheminement est le fait de forces extérieures :
Aujourd'hui je retourne
À la frontière
Je dois encore traverser
C'est le vent qui me commande
Et me pousse à la frontière
Et efface le chemin
Qui disparaît derrière moi

Et, en finale, elle ajoute l'idée que ce mouvement est aléatoire : "Je suis le point noir qui avance / aux abords de la chance. "

La chanson "La marée haute " montre aussi le personnage en marche, mais insiste sur l'opposition entre la raison et les émotions, opposition qui incarne souvent le conflit chez ceux qui vivent intensément une relation amoureuse :

La route chante

Quand je m'en vais

Je fais trois pas...

La route se tait

La route est noire

À perte de vue

Je fais trois pas...

La route n'est plus

Sur la marée haute

Je suis montée

La tête est pleine

Mais le cœur n'a

Pas assez 
La fin pourrait sembler heureuse, "Je fais trois pas... / Et tu es là ", mais la répétition en finale de l'opposition " cœur/tête » revient dire la difficulté de se rencontrer dans l'harmonie.

Ce thème de la difficulté de la rencontre revient en force dans « Mon nom ». Le personnage féminin " attend là " " placée sur la route ", " chante comme une esclave ", " tremble de peur » et implore l'Autre de lui répondre et de venir à sa rencontre :

Tu es venu si proche

Tu peux t'approcher encore

[...]

Les choses s'éloignent

Les unes des autres

La tête des mains

Et les mains du cœur

J'ai besoin de toi

Maintenant

Pas un jour

Quand je serais [sic] prête

Descends sur la route

Descends sur la route

Mon nom mon nom

Rien n'est plus pareil

Je ne retournerais [sic] pas

Par le même chemin

Or la rencontre semble de plus en plus improbable dans « Pour la fin du monde ou le nouvel an » où la narratrice attend le retour de l'amant pour le " tuer » ou le " libérer ». Les images de son "squelette qui danse ", de "navires de guerre » de "pluie de cendres" annoncent sans l'ombre d'un doute la mort de la relation amoureuse. Elle affirme qu'elle "n'a plus peur de dire que l'amour est parti ", elle "sort le rencontrer ", mais seulement "à mi-chemin " et avoue qu'elle l'a trahi. Le refrain, répété trois fois, vient clore le débat et donner les raisons de cet échec : «Et après... et après sept ans d'exil / Pour t'avoir tant / Tant menti ». Cette chanson est à rapprocher de "La confession " dans laquelle la narratrice répète qu'elle "n’a pas peur de dire que je t'ai trahi " et "Je n'ai pas peur de dire que j'ai triché" ".

Enfin, la dernière chanson de l'album prend en rétrospective une dimension prophétique. En effet, dans "Bientôt cet espace sera trop petit ", il ne s'agit plus de la difficulté de rencontrer l'Autre ou de la mort d'une relation amoureuse, mais bien de la mort de la narratrice qui dit : 
Bientôt cet espace sera trop petit

Et j'irais [sic] dehors

Sur l'immense flanc de colline

Où soufflent les vents sauvages

Et brillent les étoiles froides

Bientôt cet espace sera trop petit

Toutes mes veines et mes os

Seront brûlés jusqu'à la poussière

Tu peux me jeter

Dans un chaudron en fer noir

Et ma poussière dira

Ce que ma chair

N'a pas voulu dire

Pourtant, ces images de mort sont contrastées, voire niées, par des images de résurrection :

Bientôt cet espace sera trop petit

Et je rirai si fort

Que les murs s'écrouleront

Et puis je mourrai trois fois

Et je naîtrai encore

Dans une petite boîte

Avec une clef d'or

Et un poisson volant

Me libérera

Est-ce que celle qui toute sa vie a traversé des espaces, des États-Unis au Mexique, du Québec en France et dans les dix-sept pays où elle a donné des spectacles à guichets fermés, pourrait même réussir à traverser la frontière entre la vie et la mort ? Est-ce que son prénom tibétain ouvre à Lhasa des voies inaccessibles aux autres? Chose certaine, la fin du chemin ne semble être que le début d'un autre pour l'éternelle nomade qui chante :

Je poserai mon pied

Sur la route vivante

Et je serai portée

Jusqu'au cœur du monde 
Le portrait saisissant de cette humaine authentique brossé par le journaliste Yves Bernard résume sa démarche artistique et en souligne les aspects essentiels : la traversée des frontières et le mouvement entre le déracinement et l'enracinement.

Elle se sentait d'identité confuse, marchait seule, refusait de vivre au passé, se permettait de monter par-dessus les hautes marées, se donnait le vertige, traversait les frontières imposées, quitte à s'y brûler, mais pénétrait la vastitude de la vie, un thème central de son œuvre. Que nous aurait-elle réservé si elle ne s'était pas éteinte aussi rapidement ? Assurément toujours ce même cœur saignant, plusieurs autres révolutions sur elle-même et l'ouverture constante de cette route hors norme à suivre pour ces créateurs déracinés ou réenracinés. (Bernard 2010, A8)

Car, comme elle l'écrit elle-même dans La route chante : «Une chanson est vivante. Elle est vivante parce que quelqu'un a rêvé très fort pour la créer. " (De Sela 2008, 49) Alors pour Lhasa de Sela, comme le rêve et la route, la chanson est vivante, ne meurt jamais. Et la traversée des espaces géographiques, culturels et musicaux s'accompagne aussi d'une fusion des genres chez cette créatrice qui avoue :

J'ai toujours des images de peintures en tête. Des images incroyables... [...] D'ailleurs, chaque chanson a pour moi son univers visuel, ses couleurs. Écrire une chanson, c'est comme trouver une trame, un fil magique qu'on suit, et ce sont ces univers visuels qui me guident. (De Sela 2008, 74)

Force est de constater que Lhasa de Sela a su trouver ce fil magique ; ses chansons continuent de nous envoûter comme une lumière immortelle.

\section{Notes}

1 Les textes des chansons se trouvent dans le livret accompagnant le CD.

\section{Bibliographie}

Bernard, Yves : "Lhasa de Sela 1972-2010 - départ prématuré d'une artiste marquante ». In : Le Devoir (04.01.2010), A1 (page couverture) et A8.

Cassivi, Marc : «Lumière silencieuse ». In : La Presse (05.01.2010), C1-C2.

Chartier, Daniel : «Les origines de l'écriture migrante. L'immigration littéraire au Québec au cours des deux derniers siècles ». In : Voix et Images 27,2 (2002), 303-316.

Chartrand, Luc : «Un melting-pot nommé Lhasa ». In : L'Actualité (01.01.1998), 24. 
De Sela, Lhasa : La route chante. Paris : Éditions Textuel, 2008.

De Sela, Lhasa : "Site officiel ». In : http://lhasadesela.com/biographie/ (consulté le 15.10.2017).

Lévesque, Solange : «Le pays, c'est le chemin ». In : Le Devoir (22.10.2003), B8.

Vigneault, Alexandre : «Lhasa de Sela, l'étoile filante ». In : La Presse (05.01.2010), A1 (page couverture) et C1-C2 (Cahier Arts et Spectacles).

Vigneault, Alexandre : «La face cachée de Lhasa ». In : La Presse (18.04.2009), A1 (page couverture) et C2-C3 (Cahier Arts et Spectacles).

\section{Discographie}

De Sela, Lhasa : La llorona. Les Disques Audiogramme ADCD 10101, 1997 (CD).

De Sela, Lhasa: The Living Road. Les Disques Audiogramme ADCD 10165, 2003 (CD).

De Sela, Lhasa : Lhasa. Les Disques Audiogramme ADCD 10222, 2008 (CD). 